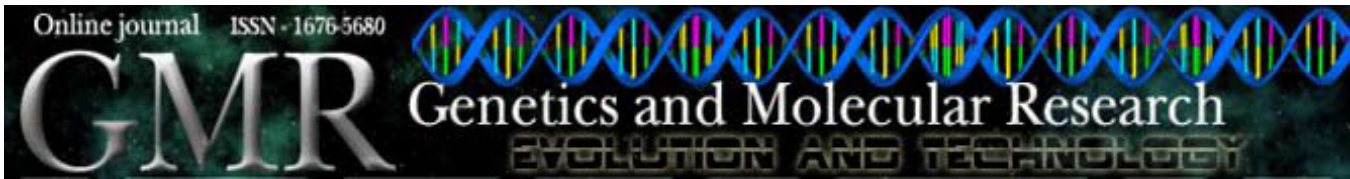

\title{
An enhancer/promoter combination strengthens the expression of blood-coagulation factor VIII in non-viral expression vectors
}

\author{
V. Picanço-Castro ${ }^{1}$, E.M.S. Russo-Carbolante ${ }^{1,2}$, A.M. Fontes ${ }^{1}$, \\ A.C. Fernandes ${ }^{1}$ and D.T. Covas ${ }^{1,3}$ \\ ${ }^{1}$ Hemocentro de Ribeirão Preto, Faculdade de Medicina de Ribeirão Preto, \\ USP, Ribeirão Preto, SP, Brasil \\ ${ }^{2}$ Departamento de Análises Clínicas Toxicológicas e Bromatológicas, \\ Faculdade de Ciências Farmacêuticas de Ribeirão Preto, USP, \\ Ribeirão Preto, SP, Brasil \\ ${ }^{3}$ Departamento de Clínica Médica, Faculdade de Medicina de Ribeirão Preto, \\ USP, Ribeirão Preto, SP, Brasil
}

Corresponding author: V. Picanço-Castro

E-mail: v.picanco@hemocentro.fmrp.usp.br

Genet. Mol. Res. 7 (2): 314-325 (2008)

Received February 25, 2008

Accepted February 27, 2008

Published April 8, 2008

\begin{abstract}
We explored the potential of fusion of hepatic locus control region 1 (HCR-1) with HCR-2 to express B-domain-deleted human factor VIII (FVIII) in four cell lines. B-domain-deleted human FVIII expression was controlled by HCR-1/HCR-2, followed by liver specific and ubiquitous promoters. Chimera enhancer HCR-1/HCR-2, followed by cytomegalovirus (CMV) promoter, gave 2-fold more FVIII expression in all cell lines (105.6 \pm 2.8 for Hek-293, $68.8 \pm 3.8$ for HepG2, $34.8 \pm 1.3$ for $\mathrm{CHO}$, and $27.2 \pm$ $1.6 \mathrm{ng} \cdot \mathrm{mL}^{-1} \cdot 10^{6}$ cells $^{-1}$ for L.N.) when compared to the vector with CMV alone $(54.8 \pm 3.3$ for Hek-293, $32.4 \pm 1.2$ for HepG2, $18.6 \pm$ 1.1 for $\mathrm{CHO}$, and $10.1 \pm 1.7 \mathrm{ng} \cdot \mathrm{mL}^{-1} \cdot 10^{6}$ cells $^{-1}$ for L.N.). Elongation factor 1- $\alpha$ gene and human CMV promoters were more efficient than the promoters from the human $\alpha$-1-antitrypsin gene, and fviii
\end{abstract}


was less efficient in hepatic cell lines. HCR-1/HCR-2, followed by strong promoters, increases FVIII expression in vitro. Our results underscore the importance of cis sequences for enhancing in vitro FVIII expression; this may be helpful for designing new strategies to improve heterologous expression systems.

Key words: Coagulation; Human factor VIII; Plasmid vector; Enhancer HCR-1 and HCR-2; Liver specific promoter; Mammalian cell lines

\section{INTRODUCTION}

Factor VIII (FVIII) is a plasma glycoprotein that participates in the blood clotting cascade as a co-factor of factor IX in the activation of factor X. Hemophilia A is an X-linked bleeding disorder that affects 1 in 5000 males, resulting in defective or deficiency of FVIII (Gitschier et al., 1984). However, in severe cases, hemophilic patients present $<0.01$ IU FVIII/ $\mathrm{mL}$ blood, which results in spontaneous and prolonged bleeding in joints, muscles, soft tissues, and internal organs, with a need for exogenous replacement by either plasma- or recombinantderived FVIII preparations (Hoyer, 1994; Antonarakis et al., 1995).

Intravenous administration of plasma-derived or recombinant FVIII (rFVIII) protein is currently the most accepted treatment for bleeding episodes in hemophilic patients. Although, this replacement therapy is effective, it has several drawbacks. These include limited availability, high costs, short half-life of the FVIII protein in vivo, risk of blood-borne transmissible diseases, and development of antibodies against FVIII in about 20\% of the patients (Hoyer, 1994).

rFVIII has many advantages over FVIII concentrates, including a high degree of purity and no risk of human-derived viral transmission. FVIII expression in vitro is often inefficient; using the same expression systems, the expression levels achieved in Chinese hamster ovary $(\mathrm{CHO})$ and COS cells are two to three times lower than for similar proteins (Kaufman et al., 1988, 1989). In contrast to full-length FVIII, in vitro expression of B-domain-deleted FVIII (FVIIIDB) results in 2- to 10-fold higher production levels (Meulien et al., 1988; Pittman et al., 1993; Lind et al., 1995). Although, some strategies have been tested to improve expression of the FVIII gene, such as deletion of the B-domain, the results have not been as satisfactory as expected. Modifications in the rFVIII molecule have been made to increase mRNA levels, improve secretion efficiency and increase the rate of thrombin activation (Fang et al., 2007).

The liver is the main physiological site of FVIII synthesis, although biologically active FVIII can be synthesized in other tissues (Wion et al., 1985). Transcriptional targeting to the liver can be achieved by using liver-specific promoters. The murine albumin promoter-enhancer has been shown to drive factor IX expression in recipient animals for up to six months (Mingozzi et al., 2003). The human $\alpha$-1-antitrypsin (hAAT) promoter has proven to be more potent in hepatocytes than the murine promoters for albumin, RNA polymerase II, and phosphoenolpyruvate carboxykinase (Hafenrichter et al., 1994a; Lam et al., 2007). In addition, transcription of hAAT from the hAAT promoter can be sustained in vivo at high levels for over 40 weeks (Hafenrichter et al., 1994b). Enhancer elements, such as the HCR-1 (hepatic locus control region 1) of gene locus ApoE, have also been found to direct expression in liver cells. This region can keep the chromatin transcription- 
ally active. Moreover, HCR-1 has a matrix attachment region and liver-specific enhancer elements, which can significantly increase gene expression (Simonet et al., 1993). Allan et al. (1995) demonstrated that a second region HCR (HCR-2) is located $27 \mathrm{~kb}$ downstream of HCR-1; it functions as a hepatic enhancer and has activity similar to HCR-1.

We analyzed the efficiency of various constructions to express FVIII. Five different constructions containing FVIIIDB were generated with an expression cassette that includes HCR-1 and HCR-2 linked by tata box followed either by liver-specific promoters (hAAT or physiological FVIII promoter) or by ubiquitous promoters (cytomegalovirus, CMV or elongation factor 1- $\alpha, E F 1-\alpha$ ). This is the first time that expression of FVIII has been demonstrated using plasmid vectors containing HCR-1 and HCR-2 combined with different promoters.

\section{MATERIAL AND METHODS}

\section{Plasmid constructions}

In order to generate the FVIII plasmid vectors, full-length FVIII-cDNA from ATCC (accession number K01740) was used as a template for the polymerase chain reaction (PCR).

pN-FVIII/HC-EGFP: the heavy chain (HC) was cloned into a pN2 plasmid vector (Clonthec, USA) in fusion with an enhanced green fluorescent protein (EGFP). The HC was amplified using sense primer 5'-GTCGACATGCAAATAGAGCTCTCCACC-3' and reverse primer 5'-GATATCTGAATTCTGGGAGAAGCTTCTTG-3'. The HC is from nucleotide 172 to 2466 (including 24 amino acids of B-domain). The amplified fragment was cloned between SalI and EcoRV sites of the multiple cloning site (MCS) of mammalian expression vector pEGFP (Clonthec, USA). The MCS region is between the CMV promoter and the EGFP.

pSecTag-FVIII/LC-c-myc: the light chain (LC) was cloned into pSecTagB in fusion with the IgK signal peptide (upstream) and c-myc (downstream). The LC was amplified using the sense primer 5'-GTAATCCAAAACCCACCAGTCTTGAAACG-3' and the reverse primer 5'-CCCTCGAGCGTAGAGGTCCTGTGCCTC-3'. The LC is from nucleotide 5140 to 7227 , including 34 amino acids of the B-domain. The fragment was cloned between EcoRV and $\mathrm{XhoI}$ sites of MCS of mammalian expression vector SecTagHygroB (Invitrogen, USA). The MCS region is between the IgK signal peptide for protein secretion and the c-myc gene.

pcDNA3.1-HCR-1-2-FVIIIDB: The FVIIIDB was cloned into a pcDNA3.1 plasmid under control of the CMV promoter. The deletion of nucleotides 2428-5067 from the FVIII full-length cDNA results in a functional FVIII protein. These nucleotides code for amino acids 760 to 1639 , which represent part of the B-domain of the protein. HCR-1 and HCR-2 were obtained from the vector pBS-HCR-1-2, kindly provided by Dr. Torsten Tonn, Institute for Transfusion Medicine and Immunohaematology, Frankfurt, Germany. To clone the region HCR-1-2 into the pcDNA3.1-cmv-FVIIIDB, the pBS-HCR-1-2 vector was digested with the enzymes EcoRV and XhoI to release the HCR-1-2 fragment. This fragment was treated with klenow enzyme to form blind ends and cloned into the vector pcDNA-cmv-FVIIIDB. This vector was previously digested with $M l u \mathrm{I}$ (restriction site located upstream to the CMV promoter) and treated with the klenow enzyme. The HCR-1-2 fragment and the vector (pcDNA-cmvFVIIIDB) were linked with T4 ligase (Invitrogen, German) to form the vector pcDNA-HCRcmv-FVIIIDB. The fragments encoding the hAAT, EF1- $\alpha$ and fviii promoters were amplified by PCR using specific primers (Table 1). 


\begin{tabular}{|c|c|}
\hline Primers & Sequence \\
\hline Fviii-MunI forward & 5'-cccaattgggetgcttcccactgataaa-3' \\
\hline Fviii-NotI reverse & 5'-gcggccgcctggagaagcaaaaggttaat-3' \\
\hline hAAT-MunI forward & 5 -cccaattgatttttccatcgggaccatc-3' \\
\hline hAAT-NotI reverse & 5 '-atgcggecgegtcctcgtccgtatttaagca-3' \\
\hline EF1- $\alpha-M u n I$ forward & 5 '-cccaattgattcaagcttcgtgaggctc- 3 ' \\
\hline EF1- $\alpha-N o t$ I reverse & 5'-atgcggecgcgttcacgacacctgaaatgg-3' \\
\hline
\end{tabular}

The amplified products were digested with MunI and Not I and cloned into pcDNA3.1HCR-FVIIIDB previously digested with the same restriction enzymes (MunI and NotI excise the original CMV of the pcDNA3.1 vector). For the fviii promoter, a minimal sequence consisting of nucleotides -44 to +148 was found to retain the wild-type transcriptional activity in human hepatocellular carcinoma (HepG2) cells (McGlynn et al., 1996). This promoter was used to form vector pcDNA-HCR-fviii-FVIIIDB. For the hAAT promoter, the main part of the promoter sequence that included the hepatocyte-specific regulatory response elements was known to be nucleotides -488 to +20 . This region was used to generate vector pcDNA-HCRhAAT-FVIIID (Mendel et al., 1991; Kalsheker et al., 2002). The fviii and hAAT promoters were generated by PCR from human embryonic kidney epithelial (Hek-293) cell genomic DNA. A fragment encoding the EF1- $\alpha$ promoter was generated by PCR from the plasmid pEFIRES-P to generate pcDNA-HCR-EF1- $\alpha$-FVIIIDB. The identities and correct orientation of the promoter sequences were confirmed by sequencing. Figure 1 shows the schematic representation of all constructions.

\begin{tabular}{|c|c|c|c|c|c|}
\hline pN-FVIII/HC-EGFP & CMV & FVIII-HC & \multicolumn{2}{|c|}{ EGFP } & \\
\hline pSecTag-FVIII/LC-c-myc & CMV & FVIII-LC & \multicolumn{2}{|c|}{ C-MYC } & \\
\hline pcDNA3.1-CMV.FVIIIDB & CMV & \multicolumn{2}{|c|}{ FVIII-DB } & & \\
\hline pcDNA3.1-HCR-1-2.CMV-FVIIIDB & HCR-1-2 & tata & HCR-2 & CMV & FVIIIDB \\
\hline pcDNA3.1-HCR-1-2.fviii-FVIIIDB & HCR-1-2 & tata & HCR-2 & fviii & FVIIIIDB \\
\hline pcDNA3.1-HCR-1-2-EF1- $\alpha$-FVIIIDB & HCR-1-2 & tata & HCR-2 & EF1- $\alpha$ & FVIIIDB \\
\hline pcDNA3.1-HCR-1-2-hAAT-FVIIIDB & HCR-1-2 & tata & HCR-2 & hAAT & FVIIIDB \\
\hline
\end{tabular}

Figure 1. Schematic representation of plasmid vectors expressing factor VIII (FVIII) cDNA under control of liverspecific promoters and enhancers. pN-FVIII/HC-EGFP contains the human cytomegalovirus (CMV) immediate-early gene promoter and the FVIII heavy chain $(\mathrm{FVIII} / \mathrm{HC})$ in fusion with enhanced green fluorescent protein (EGFP). pSecTag-FVIII/LC-c-myc contains the CMV promoter, the IgK sinal peptide, FVIII light chain (FVIII/LC) and c-myc tag. pcDNA3.1-CMV-FVIIIDB contains the CMV promoter and FVIII with B-domain deleted (FVIIIDB). The constructions pcDNA3.1-HCR-1-2-CMV-FVIIIDB, pcDNA3.1-HCR-1-2-fviii-FVIIIDB, pcDNA3.1-HCR-1-2-hAAT-FVIIIDB, and pcDNA3.1-HCR-1-2-EF1- $\alpha$-FVIIIDB contain the FVIIIDB under the control of enhancer regions HCR-1 and HCR-2 (HCR-1-2), followed by promoters CMV, fviii, hAAT, and EF1- $\alpha$, respectively. 


\section{Cells}

All cell lines were obtained as seed stocks from the German Collection of Microorganisms and Cell Cultures, Braunschweig, Germany (DSMZ; www.dsmz.de) or from the ATCC (www.atcc.org), Cell Biology Collection. They were further expanded and stored in liquid nitrogen. Hek-293 (DSMZ ACC 305), CHO (DSMZ ACC 110), HepG2 (DSMZ ACC 180), and L.N. 4159 (ATCC CRL-10998) were cultured in Dulbecco's modified Eagle's medium (Invitrogen, Germany), supplemented with 2 mM GlutaMAX ${ }^{\mathrm{TM}}-\mathrm{I}$ (Invitrogen) and with $10 \%$ heat-inactivated fetal bovine serum (Biochrom AG, Berlin, Germany). All cultures were grown as monolayers at $37^{\circ} \mathrm{C}$ in $5 \% \mathrm{CO}_{2}$ and saturated humidity.

\section{Plasmid transfections}

Transfections were performed in a six-well plate at a cell confluence of around 70\%, and 19 $\mu \mathrm{L}$ Lipofectamine 2000 (Invitrogen), $1.5 \mu \mathrm{g}$ endotoxin-free plasmid DNA (purified with QIAgen Plasmid Kit, called Endo free maxi), and $1 \mathrm{~mL}$ serum-reduced medium (OptiMEM, Gibco) were mixed (transfection mix) in each well. After a 30-min incubation, the transfection mix was added to the cells, which were incubated overnight (up to $14 \mathrm{~h}$ ). Transfection was stopped by collecting the mix and incubating the cells with normal medium. For co-transfection of FVIII-HC and FVIII-LC, $1 \mu \mathrm{g} / \mathrm{mL}$ calcium ionophore, $0.1 \mu \mathrm{g} / \mathrm{mL}$ phorbol 12 -myristate 13 -acetate (PMA) and $0.05 \mu \mathrm{g} / \mathrm{mL}$ von Willebrand factor (vWF) were added to the culture medium $12 \mathrm{~h}$ after transfection.

\section{RT-PCR analysis}

Total RNAs $(2 \mu \mathrm{g})$ from each sample were reverse transcribed using random primers and Superscript II Reverse Transcriptase (RT, Gibco BRL). Then, cDNAs $(2 \mu \mathrm{L})$ were used as a template for subsequent PCR experiments. The primers used to amplify a 526-bp fragment of FVIII-HC were 5'-CTTGGACAGTTTCTACTG-3' (forward) and 5'-GACGGACATCAGTGATTC-3' (reverse); the primers used to amplify a 974-bp fragment of FVIII-LC were 5'-CATGTGTTCACTGTACG-3' (forward) and 5'-GGTGTGAAGGAGTCTTG-3' (reverse). A 517-bp fragment of human $\beta$-actin was amplified as an internal control. P1F: 5'-GGCATCGTGATGGACTCCG-3'; P1R: 5'-GCTGGAAGGTGGACAGCGA-3'. All primers used in RT-PCR were located in different exons. The PCR profile was $94^{\circ} \mathrm{C}$ for $2 \mathrm{~min}$, followed by 34 cycles of $94^{\circ} \mathrm{C}$ for $30 \mathrm{~s}, 55^{\circ} \mathrm{C}$ for 30 $\mathrm{s}$ and $72^{\circ} \mathrm{C}$ for $60 \mathrm{~s}$, with a final extension step at $72^{\circ} \mathrm{C}$ for $5 \mathrm{~min}$. The PCR products were then separated by $1 \%$ agarose gel electrophoresis and gene expression was analyzed.

\section{Immunocytochemical analysis of intracellular FVIII}

The FVIII-HC was fused with EGFP. Expression of EGFP in transfected cell lines was examined on an Axioskop 2.0 Zeiss microscope (Germany) equipped with an Axiocam camera (Zeiss). The FVIII-LC was fused with c-myc. Expression of c-myc was detected with an anti-cmyc monoclonal antibody (Invitrogen, USA) and a secondary Cy3-conjugated anti-mouse IgG (Chemicon, USA). The cells were washed with phosphate-buffered saline (PBS), pH 7.2, and fixed with $2 \%$ paraformaldehyde, containing $0.3 \%$ Triton $\mathrm{X}-100$, for 10 min at $37^{\circ} \mathrm{C}$; PBS washings were done more three times. The cells were left in contact with blocking solution ( $2 \%$ bovine 
serum albumin and 5\% goat serum in PBS) for $1 \mathrm{~h}$ at room temperature, and then incubated for 1 $\mathrm{h}$ at $37^{\circ} \mathrm{C}$ in primary antibody diluted in blocking solution (anti-c-myc $=10 \mu \mathrm{g} / \mathrm{mL}$ ). Following incubation with the primary antibody, the cells were washed four times with PBS, incubated for $1 \mathrm{~h}$ at $37^{\circ} \mathrm{C}$ with the secondary antibody $(1 \mu \mathrm{g} / \mathrm{mL}$ in blocking solution), and washed again with PBS. Cover slips were mounted in $1 \mathrm{mg} / \mathrm{mL} \rho$-phenylenediamine in $90 \%$ glycerol and observed using epifluorescence microscopy (Axioskop 2, Carl Zeiss).

\section{FVIII activity quantification}

Supernatants were harvested and analyzed for FVIII expression using a two-stage chromogenic assay (Immuno GmbH, Germany). The FVIII concentrations are given as IU, as defined by the World Health Organization; $1 \mathrm{IU} / \mathrm{mL}$ FVIII is equivalent to $200 \mathrm{ng} / \mathrm{mL}$.

\section{RESULTS}

\section{The comparison of FVIII-HC and FVIII-LC cloned in separated plasmids with FVIIIDB vector}

FVIII protein production by co-transfection of pN-FVIII/HC-EGFP and pSecTag-FVIII/ LC-c-myc was compared with the production by transfection of pcDNA3.1-FVIIIDB. pcDNA3.1FVIIIDB is a hybrid molecule of $\mathrm{HC}$ and LC joined by a B-domain fragment of 14 amino acids. The FVIIIDB gave the highest expression levels in all transfected cells. The co-transfection of FVIII-LC and FVIII-HC produced very low FVIII activity in culture. However, when the co-transfected cells were treated with PMA/calcium ionofore/vWF FVIII activity rose in all transfected cells (Figure 2). However, the highest FVIII levels were found in the cells transfected with pcDNA3.1-FVIIIDB.

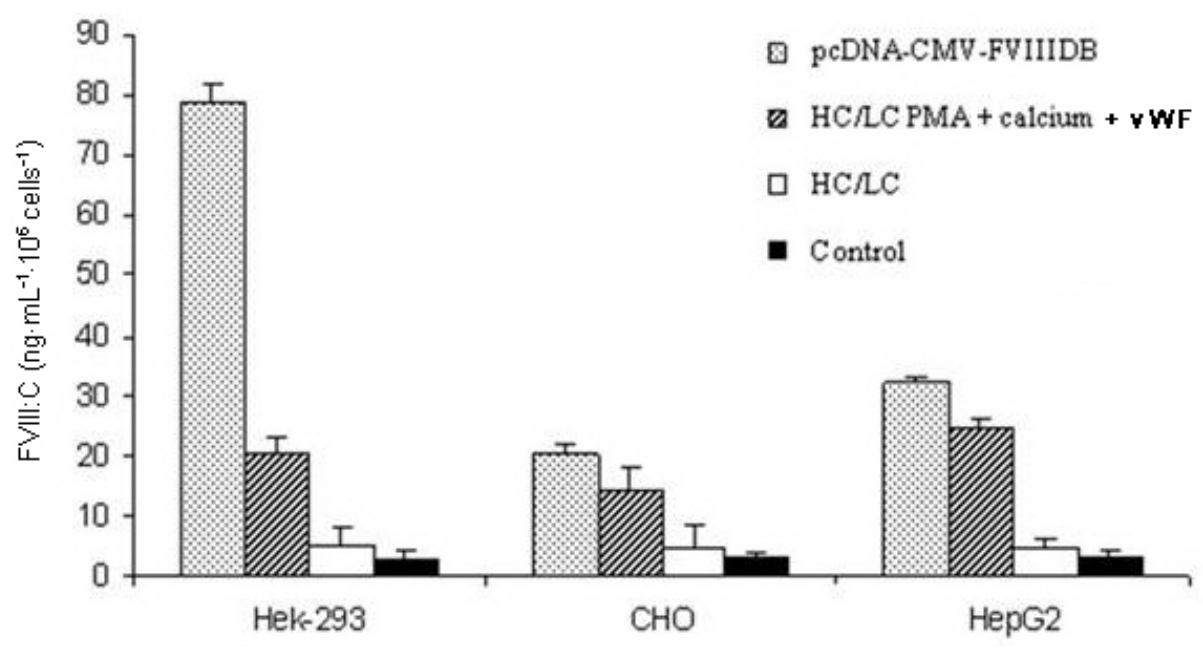

Figure 2. Comparison of FVIII activity between transient co-transfection of FVIII-HC/LC and FVIIIDB. Biological activity of FVIII secreted was performed on conditioned medium $48 \mathrm{~h}$ post-transfection. Hek-293 = human embryonic kidney epithelial cells; $\mathrm{CHO}=$ Chinese hamster ovary cells; HepG2 = human hepatocellular carcinoma cells; PMA = phorbol 12-myristate 13-acetate; vWF = von Willebrand factor. For other abbreviations, see legend to Figure 1. 


\section{Detection of FVIII-HC and FVIII-LC by RT-PCR and immunocytochemistry}

RT-PCR was used to detect FVIII-HC and FVIII-LC mRNA in low level FVIII activity co-transfected cells. All co-transfected cell lines had mRNA of both chains (Figure 3), and immunocytochemistry was used to detect intracellular FVIII protein. The cotransfected cells with FVIII-HC and FVIII-LC without adding PMA, calcium ionophore and vWF were analyzed. FVIII-LC was detected with an anti-c-myc monoclonal antibody and secondary Texas Red-conjugated anti-mouse IgG, and FVIII-HC was in fusion with EGFP. Figure 4 shows a fluorescent microscope image of CHO, Hek-293 and HepG2 cells expressing FVIII-HC and FVIII-LC $48 \mathrm{~h}$ after transfection. Immunocytochemistry showed evidence of intra-cellular co-localization of both FVIII chains in the cell. This suggests that these co-transfected cells produce the FVIII chains, but at low quantities. When the cells were treated with PMA/calcium ionofore/vWF, the FVIII was detectable in the supernatants of the cell lines.

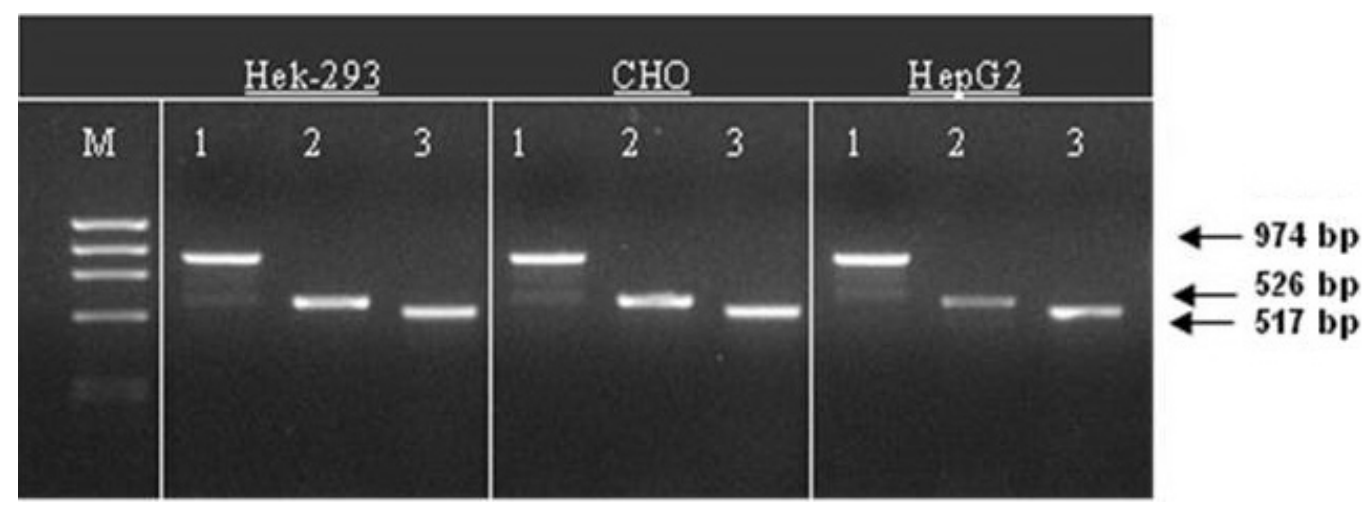

Figure 3. Heavy and light chain of FVIII is expressed in Hek-293, CHO and HepG2 co-transfected cell lines. Total RNA of these mammalian cell lines was isolated $48 \mathrm{~h}$ after co-transfection with DNA plasmids containing heavy and light chains of FVIII. The cDNA was prepared and analyzed for sequences covering the heavy and light chains of the FVIII and $\beta$-actin, as indicated. The PCR products were separated on $1 \%$ agarose gels stained with ethidium bromide and visualized in UV light. Line 1, Sequences covering the heavy chain of rFVIII; lane 2, sequences covering the light chain of rFVIII; line 3, sequences covering the $\beta$-actin transcript. Molecular size markers $(M)$ are shown to the left $(\phi X 174$ RF DNA/HaeIII - fragments: $1.3 ; 1.0 ; 0.8 ; 0.6 ; 0.3$; $0.2 \mathrm{~kb})$. For abbreviations, see legend to Figure 2.

\section{Evaluation of promoter activity by measuring FVIII and activity}

The potential of liver enhancer/promoters for producing FVIII in liver cells was also examined. We profiled the activity of enhancer/liver-promoters in comparison with enhancer/ubiquitous-promoters for expression of FVIII in hepatic and non-hepatic cell lines. We used vector pcDNA3.1-FVIIIDB, which gives the highest FVIII levels. The chimera enhancer region HCR-1-2 was cloned in this vector, followed by hAAT, fviii, EF1- $\alpha$, and CMV promoter (Figure 1). 

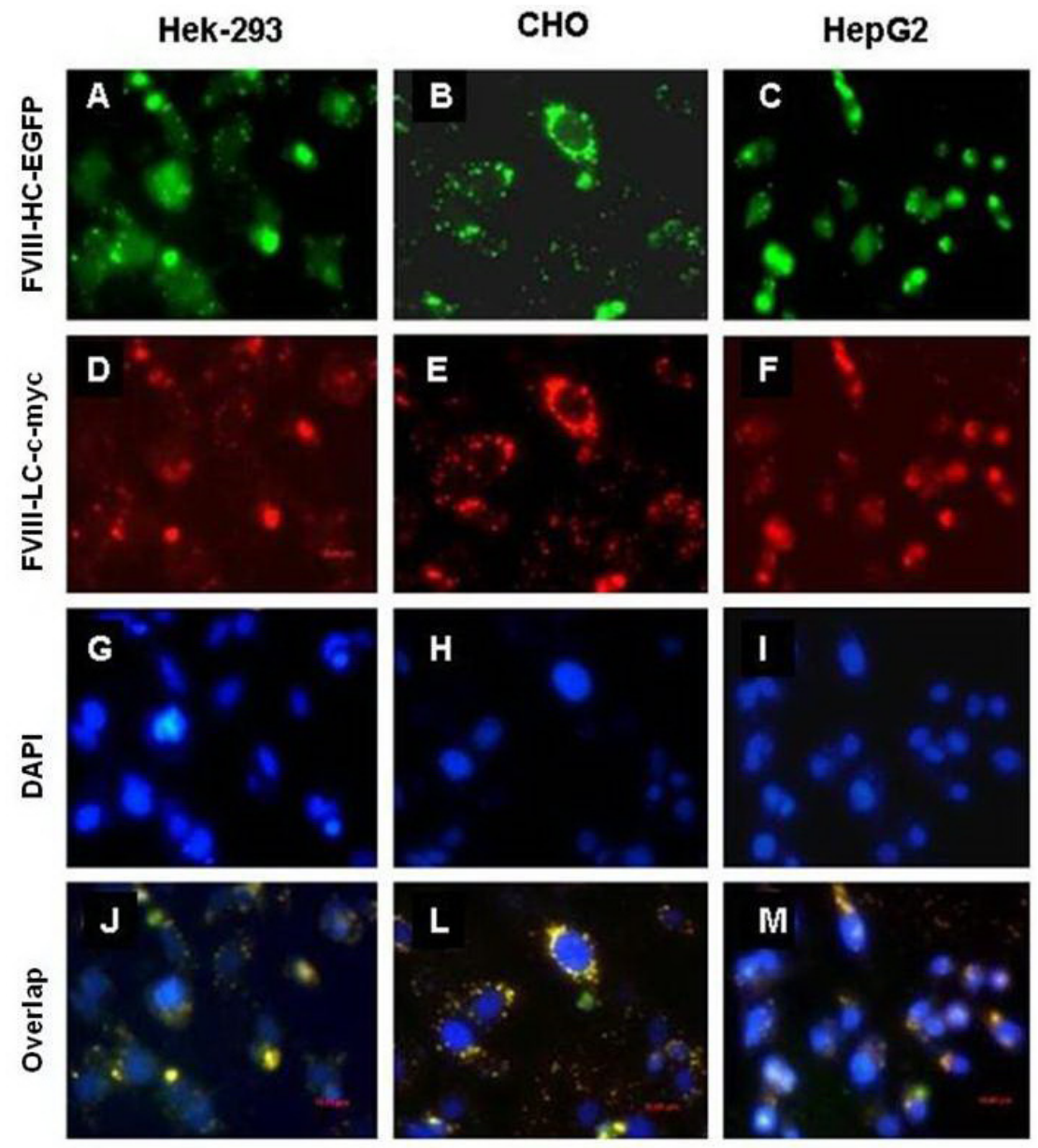

Figure 4. Intracellular localization of FVIII heavy and light chains in Hek-293, CHO and HepG2 cells. Images of epifluorescence microscopy showing triple fluorescent labeling: heavy chain-EGFP (A, B and C); the c-myc in fusion with light chain was labeled with the secondary antibody conjugated with Cy-3 (D, E and F), and the chromatin was stained blue with the fluorescent stain DAPI $(\mathrm{G}, \mathrm{H}$ and $\mathrm{I})$. In the region where the two chains overlapped, the resulting color is yellow ( $\mathrm{J}, \mathrm{L}$ and $\mathrm{M})$. These cells were fixed $48 \mathrm{~h}$ after transfection. DAPI = 4,6-diamidino-2-phenylindole. For other abbreviations, see legends to Figures 1 and 2.

FVIII activity was measured to profile the activity of generalized and liver-specific promoters for expression of FVIII in different cell lines. The CMV and EF1- $\alpha$ promoters drive expression of a variety of proteins, and they are not tissue-specific. In contrast, promoters fviii and hAAT are controlled by liver-specific factors. FVIII activity was measured in $10^{6}$ cells after a 48 -h cultivation for $48 \mathrm{~h}$. The results demonstrated different levels of expression (Figure 5). The efficiency of each promoter and cell line differed markedly. The combination HCR-1-2/EF1- $\alpha$ expression constructs gave the best yield (184.4 \pm 7.1 for Hek-293, $133.6 \pm$ 3.0 for HepG2, $38.4 \pm 1.9$ for $\mathrm{CHO}$, and $20.8 \pm 1.7 \mathrm{ng} \cdot \mathrm{mL}^{-1} \cdot 10^{6}$ cells for L.N.), followed by 
HCR-1-2/CMV (105.6 \pm 2.8 for Hek-293, $68.8 \pm 3.8$ for HepG2, $34.8 \pm 1.3$ for CHO, and 27.2 $\pm 1.6 \mathrm{ng} \cdot \mathrm{mL}^{-1} \cdot 10^{6}$ cells for L.N.). Both HCR-1-2/EF1- $\alpha$ and HCR-1-2/CMV cassette expression contributed to increasing the FVIII expression level in all cell lines.
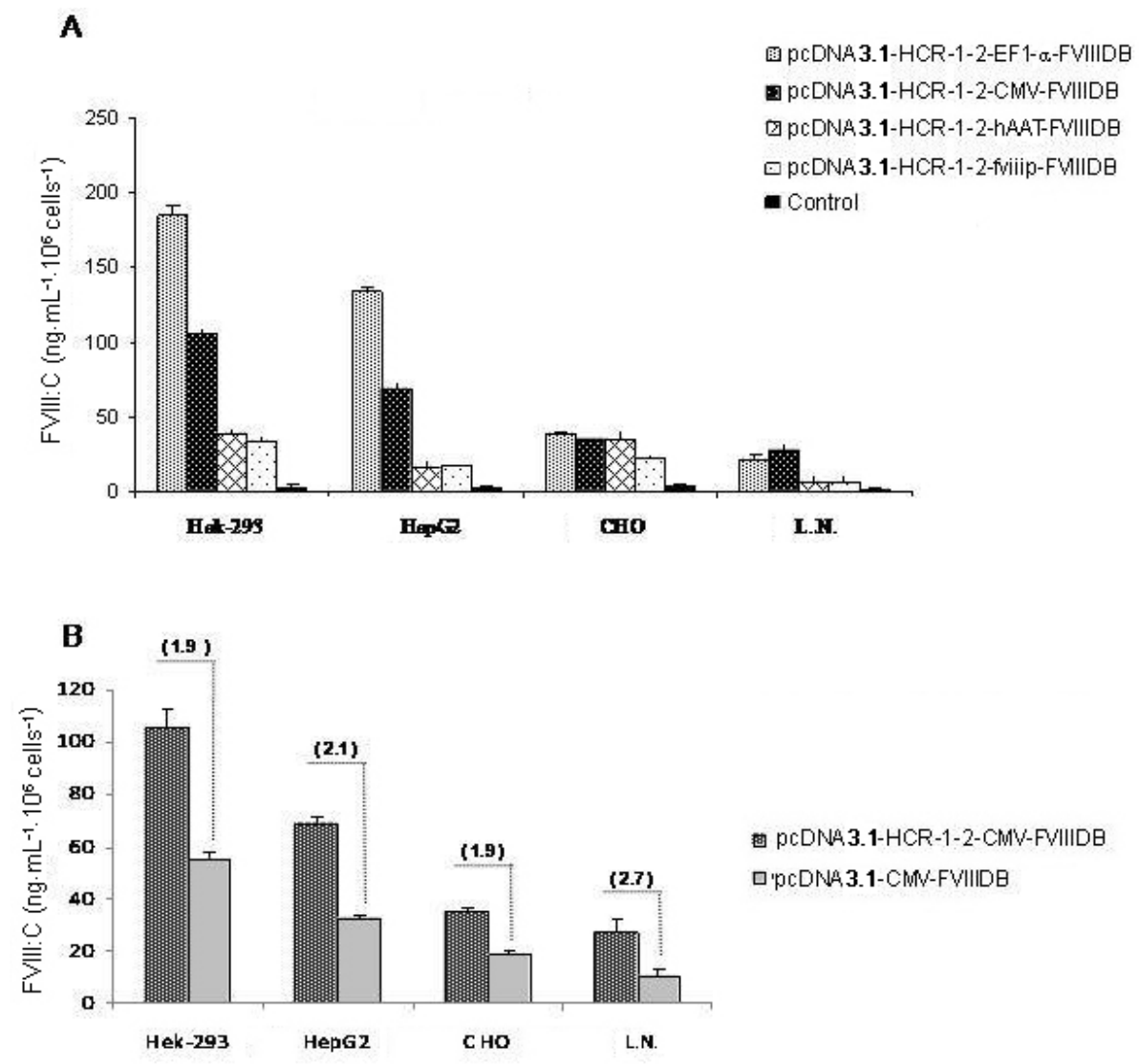

Figure 5. A. Comparison of FVIII activity (FVIII:C) in hepatic HepG2 and L.N. cell lines and non-hepatic Hek-293 and $\mathrm{CHO}$ cell lines after plasmid transfection. Cells were transfected with the vectors containing FVIIIDB under control of HCR-1-2/CMV; HCR-1-2/EF1- $\alpha$; HCR-1-2/hAAT, and HCR-1-2/fviii promoters (fviiip). B. Comparison of FVIII activity from the vectors pcDNA-HCR-1-2/CMV-FVIIIDB and pcDNA-CMV-FVIIIDB in cell lines. FVIII:C level in supernatants $48 \mathrm{~h}$ after plating out $10^{6}$ cells measured by a chromogenic assay (Immuno, Germany). The numbers in parentheses indicate how many times the FVIII activity was increased. For abbreviations, see legends to Figures 1 and 2.

The combination of enhancer HCR-1-2 with the liver-specific promoter hAAT and with the fviii promoter gave significantly lower FVIII expression (HCR-1-2/hAAt: $39 \pm 3.1$ for Hek-293, $16.6 \pm 2.2$ for HepG2, $35 \pm 1.8$ for CHO, and $6.6 \pm 1.7 \mathrm{~mL} / 10^{6}$ cells for L.N.; HCR-1-2/fviii: $33.4 \pm 2.6$ for Hek-293, $18 \pm 1.6$ for HepG2, $22.8 \pm 2.1$ for CHO, and $6.8 \pm 1.5$ $\mathrm{mL} / 10^{6}$ cells for L.N.) (Figure $5 \mathrm{~A}$ ). The FVIII activity produced by pcDNA-HCR-1-2/CMVFVIIIDB was on average two times higher than the FVIII activity produced by pcDNA-CMVFVIIIDB in all cell lines (Figure 5B). 


\section{DISCUSSION}

We used a DNA plasmid system, which is easy and fast, to probe a combination of two hepatic enhancer regions (HCR-1 and HCR-2) followed by different promoters and the recombinant protein for transient expression.

First, we used separate plasmid vectors to deliver the LC and HC of FVIII to three cell lines. In all transfected cell lines, immunocytochemistry indicated that the proteins of the two chains were co-localized intracellularly. Without PMA, calcium ionophore and vWF, FVIII was not detectable in the supernatants of these transfected cell cultures. After addition of these secretion factors, the three cell lines gave about $20 \mathrm{ng} / \mathrm{mL}$ FVIII activity; however, this value is four times lower than the FVIII activity obtained with FVIIIDB-vector transfected cells. This result indicates that PMA, calcium ionophore and vWF improve FVIII-HC and FVIII-LC expression and/or secretion. Burke et al. (1986) has shown that LC and HC cloned in identical vectors give different expression levels. The LC contains sequences that inhibit transcription, causing an imbalance in the expression of the chains, which may be the cause of the lack of activity in transfected cells (Chen et al., 2007). There are a number of factors that could account for this chain imbalance, including differences in vector gene transfer, transgene transcription, and/or protein translation and secretion.

Due to the low FVIII activity obtained with $\mathrm{HC}$ and LC vectors, we decided to modify the expression vector containing the FVIII B deleted domain (pCDNA-FVIIIDB) to obtain better FVIII expression levels. We investigated whether pCDNA-FVIIIDB together with liverspecific enhancer HCR-1-2 and different promoters could increase gene expression of FVIII in various different cell lines. The promoters were EF1- $\alpha$, CMV, hAAT, and fviii. To better focus the experiments, we used truncated hAAT and fviii promoter sequences that retained the minimal transcription initiation function (Mendel et al., 1991; McGlynn et al., 1996; Kalsheker et al., 2002). We found that all four promoters were capable of directing FVIII expression in hepatocyte cell lines, as well as in Hek-293 and CHO cells.

The EF1- $\alpha$ promoter produced the highest FVIII levels in all cell lines. By comparison, the CMV promoter had relatively little activity, and the hAAT and fviii promoters had even less.

We also found that the shortest functional sequences of the fviii and hAAT promoters were also active in the non-hepatic Hek-293 cell line. This is not surprising, in view of the fact that expression of the hAAT gene has been documented in extra-hepatic sites, including undifferentiated monocytes, macrophages, intestinal epithelial cells, and respiratory epithelium (Perlmutter et al., 1985; Molmenti et al., 1993). It is possible that when these promoters were truncated, fragments responsible for their tissue specificity were removed. A truncated hAAT promoter was shown to be equally active in hepatocytes and non-hepatic NIH3T3, HeLa and Hek-293 cells (Okuyama et al., 1996; Nash et al., 2004).

Factor VIII is mainly expressed in the liver (Wion et al., 1985; Lewis et al., 1985, 1987). However, FVIII is also expressed at moderate levels in the spleen, kidney, and lymph nodes (McGlynn et al., 1996). Transcriptional activity of both the minimal fviii promoter and the minimal hAAT promoter probably requires hepatocyte nuclear factor 1 (HNF1 $\alpha)$, and it is enhanced by the absence of its homologous isoform HNF1 $\beta$ (Mendel et al., 1991; McGlynn et al., 1996). HNF1 $\alpha$ predominates in hepatocytes and seems to be responsible for the high expression of fviii or hAAT in the liver. However, HNF1 $\alpha$ is absent in hepatoma cells (ReyCampos et al., 1991). Its absence could contribute to the low level of FVIII expression that we 
found with the fviii and hAAT promoters.

We conclude that HCR-1-2 followed by CMV promoter doubles FVIII activity levels in cell lines when compared to the CMV vector without HCR-1-2. The combination of HCR-1 and HCR-2 can help optimize in vitro rFVIII gene expression. It will be of interest to determine if the cis sequence can function in a similar manner with heterologous cDNAs.

\section{ACKNOWLEDGMENTS}

Financial support from FAPESP, CNPq and FINEP is gratefully acknowledged. The authors thank Dr. Torsten Tonn (Institute for Transfusion Medicine and Immunohaematology, Frankfurt, Germany) and Stefan Heinz for their kind help with this project.

\section{REFERENCES}

Allan CM, Walker D and Taylor JM (1995). Evolutionary duplication of a hepatic control region in the human apolipoprotein E gene locus. Identification of a second region that confers high level and liver-specific expression of the human apolipoprotein E gene in transgenic mice. J. Biol. Chem. 270: 26278-26281.

Antonarakis SE, Kazazian HH, Gitschier J, Hutter P, et al. (1995). Molecular etiology of factor VIII deficiency in hemophilia A. Adv. Exp. Med. Biol. 386: 19-34.

Burke RL, Pachl C, Quiroga M, Rosenberg S, et al. (1986). The functional domains of coagulation factor VIII:C. J. Biol. Chem. 261: 12574-12578.

Chen L, Zhu F, Li J, Lu H, et al. (2007). The enhancing effects of the light chain on heavy chain secretion in split delivery of factor VIII gene. Mol. Ther. 15: 1856-1862.

Fang H, Wang L and Wang H (2007). The protein structure and effect of factor VIII. Thromb. Res. 119: 1-13.

Gitschier J, Wood WI, Goralka TM, Wion KL, et al. (1984). Characterization of the human factor VIII gene. Nature 312 : 326-330.

Hafenrichter DG, Wu X, Rettinger SD, Kennedy SC, et al. (1994a). Quantitative evaluation of liver-specific promoters from retroviral vectors after in vivo transduction of hepatocytes. Blood 84: 3394-3404.

Hafenrichter DG, Ponder KP, Rettinger SD, Kennedy SC, et al. (1994b). Liver-directed gene therapy: evaluation of liver specific promoter elements. J. Surg. Res. 56: 510-517.

Hoyer LW (1994). Hemophilia A. N. Engl. J. Med. 330: 38-47.

Kalsheker N, Morley S and Morgan K (2002). Gene regulation of the serine proteinase inhibitors alpha1-antitrypsin and alpha1-antichymotrypsin. Biochem. Soc. Trans. 30: 93-98.

Kaufman RJ, Wasley LC and Dorner AJ (1988). Synthesis, processing, and secretion of recombinant human factor VIII expressed in mammalian cells. J. Biol. Chem. 263: 6352-6362.

Kaufman RJ, Wasley LC, Davies MV, Wise RJ, et al. (1989). Effect of von Willebrand factor coexpression on the synthesis and secretion of factor VIII in Chinese hamster ovary cells. Mol. Cell Biol. 9: 1233-1242.

Lam PY, Sia KC, Khong JH, De Geest B, et al. (2007). An efficient and safe herpes simplex virus type 1 amplicon vector for transcriptionally targeted therapy of human hepatocellular carcinomas. Mol. Ther. 15: 1129-1136.

Lewis JH, Bontempo FA, Spero JA, Ragni MV, et al. (1985). Liver transplantation in a hemophiliac. N. Engl. J. Med. 312: 1189-1190.

Lewis JH, Bontempo FA, Cornell F, Kiss JE, et al. (1987). Blood use in liver transplantation. Transfusion 27: 222-225.

Lind P, Larsson K, Spira J, Sydow-Backman M, et al. (1995). Novel forms of B-domain-deleted recombinant factor VIII molecules. Construction and biochemical characterization. Eur. J. Biochem. 232: 19-27.

McGlynn LK, Mueller CR, Begbie M, Notley CR, et al. (1996). Role of the liver-enriched transcription factor hepatocyte nuclear factor 1 in transcriptional regulation of the factor VIII gene. Mol. Cell Biol. 16: 1936-1945.

Mendel DB, Hansen LP, Graves MK, Conley PB, et al. (1991). HNF-1 alpha and HNF-1 beta (vHNF-1) share dimerization and homeo domains, but not activation domains, and form heterodimers in vitro. Genes Dev. 5: 1042-1056.

Meulien P, Faure T, Mischler F, Harrer H, et al. (1988). A new recombinant procoagulant protein derived from the cDNA encoding human factor VIII. Protein Eng. 2: 301-306.

Mingozzi F, Liu YL, Dobrzynski E, Kaufhold A, et al. (2003). Induction of immune tolerance to coagulation factor IX antigen by in vivo hepatic gene transfer. J. Clin. Invest. 111: 1347-1356.

Genetics and Molecular Research 7 (2): 314-325 (2008)

CFUNPEC-RP www.funpecrp.com.br 
Molmenti EP, Perlmutter DH and Rubin DC (1993). Cell-specific expression of alpha 1-antitrypsin in human intestinal epithelium. J. Clin. Invest. 92: 2022-2034.

Nash KL, Jamil B, Maguire AJ, Alexander GJ, et al. (2004). Hepatocyte-specific gene expression from integrated lentiviral vectors. J. Gene Med. 6: 974-983.

Okuyama T, Huber RM, Bowling W, Pearline R, et al. (1996). Liver-directed gene therapy: a retroviral vector with a complete LTR and the ApoE enhancer-alpha 1-antitrypsin promoter dramatically increases expression of human alpha 1-antitrypsin in vivo. Hum. Gene Ther. 7: 637-645.

Perlmutter DH, Cole FS, Kilbridge P, Rossing TH, et al. (1985). Expression of the alpha 1-proteinase inhibitor gene in human monocytes and macrophages. Proc. Natl. Acad. Sci. U. S. A. 82: 795-799.

Pittman DD, Alderman EM, Tomkinson KN, Wang JH, et al. (1993). Biochemical, immunological, and in vivo functional characterization of B-domain-deleted factor VIII. Blood 81: 2925-2935.

Rey-Campos J, Chouard T, Yaniv M and Cereghini S (1991). vHNF1 is a homeoprotein that activates transcription and forms heterodimers with HNF1. EMBO J. 10: 1445-1457.

Simonet WS, Bucay N, Lauer SJ and Taylor JM (1993). A far-downstream hepatocyte-specific control region directs expression of the linked human apolipoprotein E and C-I genes in transgenic mice. J. Biol. Chem. 268: 8221-8229.

Wion KL, Kelly D, Summerfield JA, Tuddenham EG, et al. (1985). Distribution of factor VIII mRNA and antigen in human liver and other tissues. Nature 317: 726-729. 